\title{
Continuous treatment with lenalidomide in multiple myeloma: a case report
}

\author{
E. Müldür, A. Weißmann, C. Leitgeb, N. Zojer, M. Schreder, D. Heintel, H. Ludwig \\ Department of Medicine I, Center for Oncology and Hematology, Wilhelminenspital, Vienna, Austria
}

Received 21 December 2011; accepted 1 January 2012

We present the case of a 72-year-old woman diagnosed with multiple myeloma (MM) in 1992 and treated with lenalidomide plus dexamethasone (LD) as third-line therapy. The patient achieved a complete remission (CR) after 6 cycles of LD treatment, was treated with LD for almost 4 years, and has now been in CR for 5 years. This case illustrates the potential of lenalidomide for continuous treatment in MM, with side effects controllable through tailored patient management including step-wise dose adaptations.

Keywords: Continuous treatment, lenalidomide, multiple myeloma

\section{Introduction}

The introduction of thalidomide, bortezomib, and lenalidomide has fundamentally changed the treatment of multiple myeloma (MM). In one epidemiological study, the survival after relapse of MM patients diagnosed between 1997 and 2006 and treated with at least one of these novel drugs was found to be twice as long as that of patients not having received one or more of these agents (23.9 vs. 11.8 months, $p<0.001$ ) [1].

Treatment with conventional chemotherapy results in response rates of about $50 \%$, with only few patients achieving complete remission (CR) [2] and with a median survival of around 33 months [3]. Newer treatment regimens induce higher response rates [4-9], a better quality of response, and long-term disease control with favourable tolerance $[2,10]$.

Lenalidomide (Revlimid ${ }^{\circledR}$, Celgene, Summit, NJ) has a dual mode of action: it causes direct tumour cell death and it exerts important immunomodulatory effects, which likely contribute to maintaining remission $[11,12]$. In patients with relapsed or refractory $\mathrm{MM}$, lenalidomide in combination with dexamethasone has been found to significantly increase overall remission and CR rates, time to progression (TTP), duration of response, and overall survival (OS) compared to

Correspondence: Ercan Müldür, MD, Department of Medicine I, Center for Oncology and Hematology, Wilhelminenspital, 1160 Vienna, Austria.

E-mail: ercan.muelduer@wienkav.at single-agent dexamethasone [5, 8, 9]. In addition, lenalidomide is usually well tolerated, with initial myelosuppression as the clinically most relevant toxicity. Lenalidomide lacks cumulative toxicity [13], allowing for continuous treatment. In Europe, the drug was first approved in 2007.

We report on a 72-year-old patient diagnosed with MM in 1992 and treated with lenalidomide plus dexamethasone as third-line therapy. We consider this case of interest because of the rapid and complete response achieved with lenalidomide, the long treatment duration of almost 4 years, and the long duration of complete remission for the past 5 years.

\section{Case report}

The patient is a 72-year-old woman with IgG kappa MM first diagnosed in June 1992 at the age of 53 years. She presented with Salmon-Durie stage IIA. A fluorescence in situ hybridization (FISH) test performed in 2009 on stored bone marrow revealed no cytogenetic abnormalities. The patient was started on VMCP (vincristine, melphalan, prednisone, and cyclophosphamide) plus interferon- $\alpha$ and was treated for 12 cycles; thereafter, maintenance treatment with interferon- $\alpha$ was initiated and continued for a total of 11 years (Fig. 1).

In June 2004, the patient presented with progressive disease, mild lumbar pain and scoliotic changes and was enrolled into a randomized, placebo-controlled phase 3 study (MM-010, Celgene) [5] comparing lenalidomide-dexamethasone (LD) with placebo-dexamethasone therapy. Patients in the LD group were to receive lenalidomide at a dose of $25 \mathrm{mg}$ orally on days 1-21 of each 28-day cycle, combined with 40 mg of oral dexamethasone on days 1-4, 9-12, and 17-20 for the first four cycles and on days 1-4 thereafter. Patients in the control group received dexamethasone as above and placebo instead of lenalidomide. In addition, monthly pamidronate at a dose of $90 \mathrm{mg}$ was administered for 34 cycles. Response was evaluated according to the European Group for Blood and Marrow Transplantation (EBMT) criteria [14].

After 3 cycles of treatment, a greater than $50 \%$ reduction in M protein, corresponding to a partial remission (PR), was noted (Figs. 1 and 2), and this result was maintained over the next year. During this time, the dexamethasone dose was reduced twice (Fig. 2) because of side effects, particularly muscle weakness, impaired vision, constipation, bone pain, 


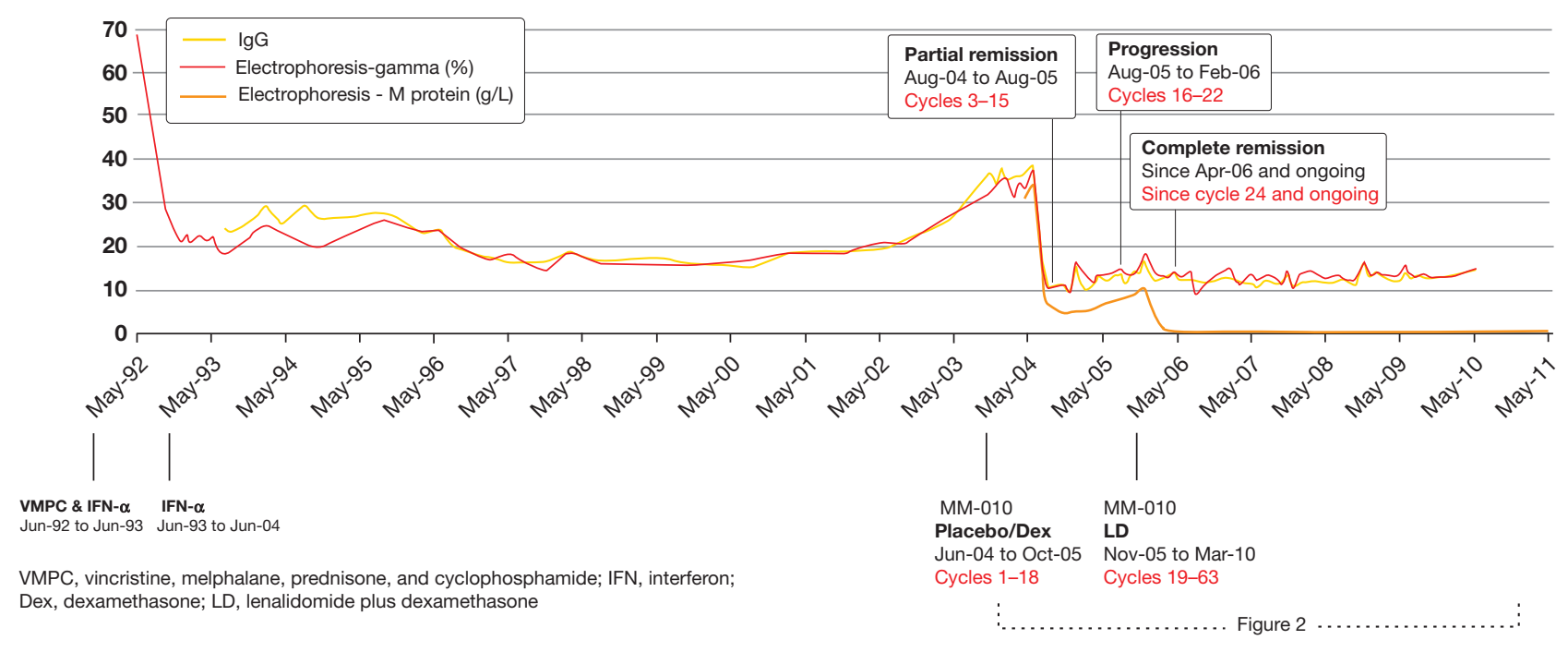

Fig. 1: Disease course based on M protein levels, 1992-2011

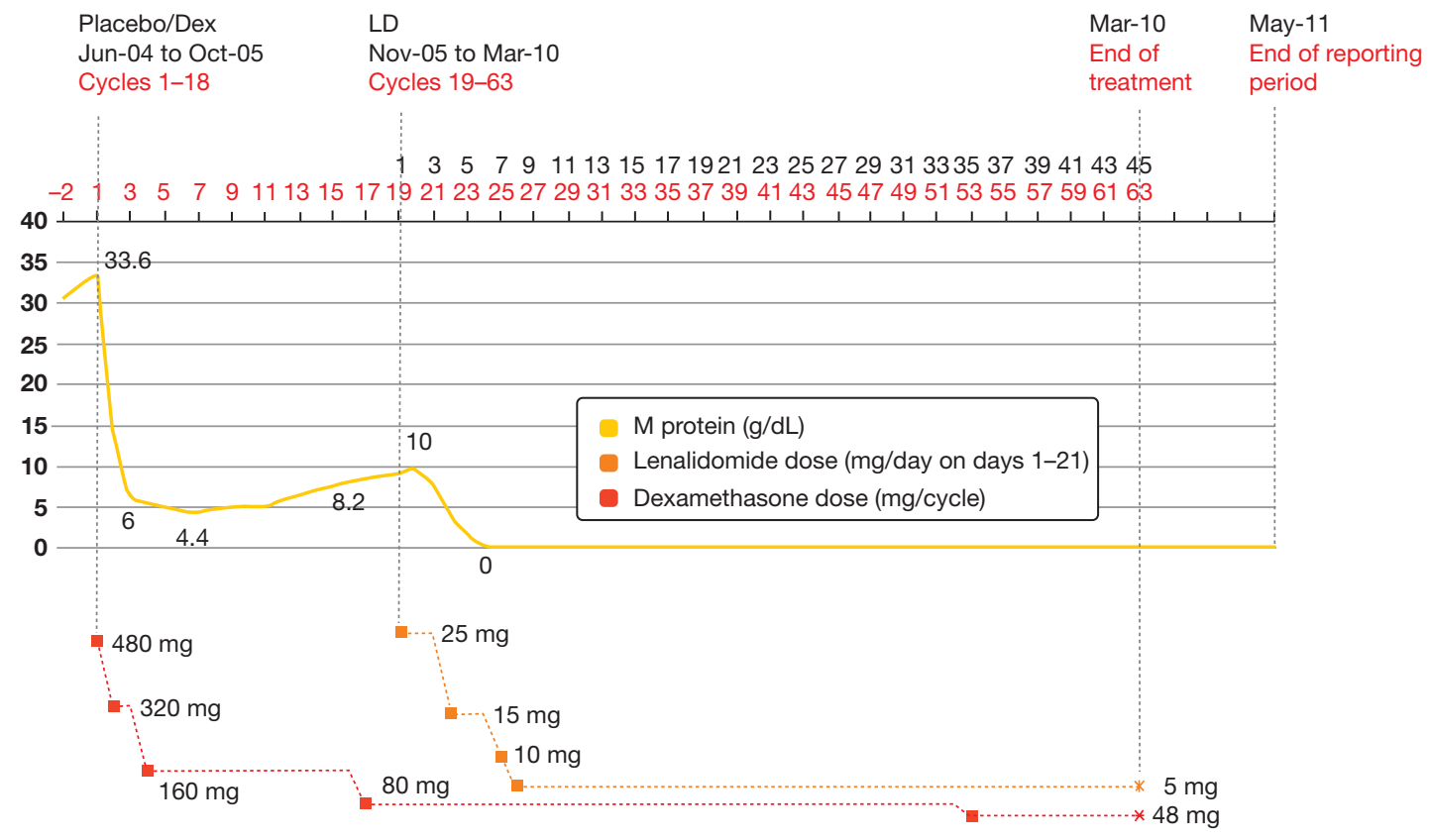

Fig. 2: $M$ protein and lenalidomide-dexamethasone (LD) doses (study cycle -2 through end of reporting period)

oedema in the lower extremities, and loss of appetite. In cycle 8 , a fracture of the first lumbar vertebral body was diagnosed.

Progressive disease was noted in cycle 17 (M protein $8.2 \mathrm{~g} / \mathrm{L}$ ), therefore the treatment code was broken, revealing that the patient had received dexamethasone only. Haemoglobin was $7 \mathrm{~g} / \mathrm{L}$, corresponding to a grade 3 toxicity for which she received 2 units of red cells. In this cycle, the dose of dexamethasone was reduced to $20 \mathrm{mg}$ on days 1-4 of each cycle because of severe leg cramps and nausea. Both symptoms subsided after the dexamethasone dose reduction.

By cycle 18, the patient's M protein had increased to $9.1 \mathrm{~g} / \mathrm{L}$, and she was crossed over to lenalidomide $25 \mathrm{mg}$ on days 1-21, with the dexamethasone dose unchanged. Apart from intermittent herpetic skin affections, the first cycles of lenalidomide were well tolerated. Cycle 22 was delayed for 1 week because of neutropenia $\left(0.9 \times 10^{9} / \mathrm{L}\right)$ and leukopenia $\left(2.7 \times 10^{9} / \mathrm{L}\right)$, corresponding to CTC grade 3 toxicity. Bullous skin disease was recorded, but subsided within 1 week, after which lenalidomide was continued at $15 \mathrm{mg}$ daily on days 1-21. At that time, the patient had already achieved a PR with a reduction of the $\mathrm{M}$ protein to $3 \mathrm{~g} / \mathrm{L}$. The lower lenalidomide dose was well tolerated, with the previously reported side effects no longer present. After 6 cycles of LD treatment, the patient was in $\mathrm{CR}$, with negative immunofixation and less than $5 \%$ of plasma cells in the bone marrow biopsy. A skeletal survey showed partial bone healing with no new bone lesions. 
LD treatment was delayed in cycles 6 and 8 (corresponding to a total of 24 and 26 cycles) because of CTC grade 3 neutropenia. This resulted in a further decrease of the lenalidomide dose to $10 \mathrm{mg}$ and, one cycle later, to $5 \mathrm{mg}$. The previously noted symptoms, such as urinary urgency and severe diarrhoea alternating with constipation, normalized completely. Continuation of therapy over the next 18 months was well tolerated without any symptoms (ECOG status: 0) through the end of therapy.

The dexamethasone dose was reduced in cycle 35 of $\mathrm{LD}$ treatment (or a total of 53 cycles) to $12 \mathrm{mg}$ on days $1-4$ of each cycle because of symptoms related to corticosteroid therapy, such as impaired vision, hypertension and polyuria. In March 2010, treatment with lenalidomide-dexamethasone was discontinued at the patient's request, after a total of 45 cycles, with 39 cycles being in complete remission and total treatment duration with lenalidomide-dexamethasone of 3 years and 9 months. At the time of this report, she continues to be in complete remission and is doing well.

\section{Discussion}

The introduction of lenalidomide has significantly expanded the treatment options in patients with MM $[5,8,9]$, both as initial and as rescue therapy. In relapsed and refractory myeloma, two pivotal phase 3 studies with $\mathrm{LD}[5,8]$ were unblinded after a median follow-up of 17.5 months because a preplanned interim analysis had shown that LD is significantly superior to dexamethasone alone [9]. Subsequently, all patients were allowed to cross over to lenalidomide-based treatment. At a median follow-up of 48 months, a significant survival benefit was maintained (38vs. 31.6 months, $p=0.045$ ), although almost half of the patients $(47.6 \%)$ on dexamethasone-placebo had been switched to lenalidomide-dexamethasone after disease progression or study unblinding.

The patient in this report had initially responded to single-agent dexamethasone but developed progressive disease after 16 cycles and was crossed over to LD, which elicited a rapid PR after only 2 treatment cycles and a CR after an additional 3 cycles, with the CR now ongoing for more than 5 years. We believe this case to be of interest because of the long survival duration and because of the rapid and complete response obtained with lenalidomide-dexamethasone even after long-term pretreatment with dexamethasone-placebo. Overall, the patient was on lenalidomide therapy for a total of 3 years and 9 months.

A subgroup analysis [15] of the two pivotal phase III studies $[5,8]$ has shown that longer durations of treatment and maintenance of best response with LD are associated with prolonged overall survival. The side effects in our patient were manageable by step-wise dose reductions, with treatment throughout the last 37 cycles consisting of $5 \mathrm{mg}$ lenalidomide daily in combination with low-dose dexamethasone. Another subgroup analysis [16] on the effects of lenalidomide dose adjustments on outcome found that patients in whom the full dose of $25 \mathrm{mg}$ daily was reduced after 12 months had a significantly longer progression-free survival than patients in whom no dose reduction was performed, supporting the assumption that patients benefit from dose reductions later in the course of treatment because lower doses improve tolerability, thereby prolonging the overall duration of treatment
Tab. 1: Summary of adverse effects during lenalidomide therapy

\begin{tabular}{lll}
\hline Adverse effect & $\begin{array}{l}\text { Induction } \\
\text { grade }\end{array}$ & $\begin{array}{l}\text { Maintenance } \\
\text { grade }\end{array}$ \\
\hline Neutropenia & 3 & 1 \\
\hline Anaemia & 2 & 1 \\
\hline Diarrhoea & 2 & 1 \\
\hline Herpetic skin affections & 2 & \\
\hline Neuropathy, sensory & & 2 \\
\hline Urinary urgency & 2 & \\
\hline Constipation & 1 & 1 \\
\hline Bullous skin disease & 1 & \\
\hline
\end{tabular}

and improving long-term outcome. Reducing the dose of dexamethasone after response to lenalidomide-based induction therapy has also been shown to improve the efficacy of LD in patients with relapsed or refractory MM [17].

Notably, the patient has now been in CR for 5 years. Obviously, she is a good-risk patient who has a good likelihood of maintaining her CR for many years or even forever. She achieved a PR after dexamethasone and a CR after subsequent lenalidomide-dexamethasone. This accords with previous reports [18] showing that the quality of the first response affects prognosis, with patients with a CR or a very good partial response (VGPR) initially having a significantly better TTP and OS than patients with PR. Of interest, the analysis [18] also found that $50 \%$ of patients who initially achieved a PR later had CR or VGPR with continued treatment, suggesting that, in the absence of disease progression or unacceptable toxicity, continuing treatment with LD provides added clinical benefits.

Lenalidomide is an ideal candidate for long-term continuous therapy in MM. It offers the convenience of oral administration, has anti-myeloma and immunomodulatory activity, provides long-term tumour suppression, and it is well tolerated even with long-term use. For example, in the two phases III studies with lenalidomide plus dexametha sone $[5,8]$, only few patients developed grade $2(1.4 \%)$ or grade $3(1.4 \%)$ peripheral neuropathy.

Dose-limiting or -delaying side effects are mainly haematological in nature and usually limited to the first 6 LD cycles. Other side effects usually are mild and easily manageable.

The probability of adverse events has been found to be higher in the first months of treatment and to decrease thereafter [19], and this was also seen in our patient (Table 1).

Throughout the second half of treatment, our patient's ECOG status improved to 0, allowing her to resume her previous working position.

Second primary malignancies (SPMs) are a published phenomenon in patients with MM [20-22] and have recently also been associated with lenalidomide maintenance treatment [23-25]. In the pivotal studies [5, 8] with lenalidomidedexamethasone for relapsed myeloma however, the incidence of invasive SPMs was within the expected background rate for this population and similar across treatment arms [26]. No 
SPM was detected in our patient treated with lenalidomide for almost 4 years.

In summary, taking into account the limitations of a single case, this report illustrates the potential of lenalidomide for long-term continuous treatment in MM, with side effects controllable through tailored patient management including step-wise dose adaptations.

\section{Conflict of interest}

The authors declare that there is no conflict of interest.

\section{References}

[1] Kumar SK, Rajkumar SV, Dispenzieri A, et al. Improved survival in multiple myeloma and the impact of novel therapies. Blood, 111: 2516-20, 2008

[2] Rajkumar SV. Treatment of myeloma: cure vs. control. Mayo Clin Proc, 83:1142-5, 2008.

[3] Kyle RA, Gertz MA, Witzig TE, et al. Review of 1027 patients with newly diagnosed multiple myeloma. Mayo Clin Proc, 78: 21-33 2003.

[4] Brenner H, Gondos A, Pulte D. Expected long-term survival of patients diagnosed with multiple myeloma in 2006-2010. Haematologica, 94: 270-5, 2009.

[5] Dimopoulos M, Spencer A, Attal M, et al. Lenalidomide plus dexamethasone for relapsed or refractory multiple myeloma. N Engl J Med, 357: 2123-32, 2007

[6] Facon T, Mary IY, Hulin C, et al. Melphalan and prednisone plus thalidomide versus melphalan and prednisone alone or reducedintensity autologous stem cell transplantation in elderly patients with multiple myeloma (IFM 99-06): a randomised trial. Lancet, 370: 1209-18, 2007.

[7] Richardson PG, Sonneveld P, Schuster MW, et al. Bortezomib or high-dose dexamethasone for relapsed multiple myeloma. N Engl J Med, 352: 2487-98, 2005.

[8] Weber DM, Chen C, Niesvizky R, et al. Lenalidomide plus dexamethasone for relapsed multiple myeloma in North America. N Engl J Med, 357: 2133-42, 2007.

[9] Dimopoulos MA, Chen C, Spencer A, et al. Long-term follow-up on overall survival from the MM-009 and MM-010 phase III trials of lenalidomide plus dexamethasone in patients with relapsed or refractory multiple myeloma. Leukemia, 23: 2147-52, 2009.

[10] Dimopoulos MA, Palumbo A, Attal M, et al. Optimizing the use of lenalidomide in relapsed or refractory multiple myeloma: consensus statement. Leukemia, 25(5): 749-60, 2011.

[11] Dimopoulos MA, Terpos E. Lenalidomide: an update on evidence from clinical trials. Blood Rev, 24: s21-s6, 2010.

[12] Davies F, Baz R. Lenalidomide mode of action: linking bench and clinical findings. Blood Rev, 24(Suppl. 1): S13-9, 2010.

[13] Zeldis JB, Knight R, Hussein M, Chopra R, Muller G. A review of the history, properties, and use of the immunomodulatory compound lenalidomide. Ann N Y Acad Sci, 1222: 76-82, 2011.

[14] Blade J, Samson D, Reece D, et al. Criteria for evaluating disease response and progression in patients with multiple myeloma treated by high-dose therapy and haemopoietic stem cell transplantation. Myeloma Subcommittee of the EBMT. European Group for Blood and Marrow Transplant. Br J Haematol, 102: 1115-23, 1998.

[15] San Miguel J, Dimopoulos M, Bravo M, Weber DM. Longer duration of treatment and maintenance of best response with lenalidomide + dexamethasone increases overall survival (OS) in patients with relapsed/refractory multiple myeloma (abstract 0949). Haematologica, 94: 382, 2009.

[16] Dimopoulos M, Hussein M, Swern A, et al. Full dose of lenalidomide for 12 months followed by a lower maintenance dose improves progression-free survival in patients with relapsed/refractory mutiple myeloma (poster). 51st ASH Annual Meeting, New Orleans, Louisiana, USA, 5-8 December 2009.

[17] San Miguel J, Dimopoulos M, Weber D, Olesnyckyj M. Dexamethasone dose adjustments seem to result in better efficacy and improved tolerability in patients with relapsed/refractory multiple myeloma who are treated with lenalidomide/dexamethasone (MM009/010 Sub-Analysis) (abstract 2712). Annual Meeting of the American Society of Hematology (ASH), Atlanta, Georgia 2007.

[18] Harousseau JL, Dimopoulos MA, Wang M, et al. Better quality of response to lenalidomide plus dexamethasone is associated with improved clinical outcomes in patients with relapsed or refractory multiple myeloma. Haematologica, 95: 1738-44, 2010.

[19] Ishak J, Dimopoulos M, Weber D, et al. Declining rates of adverse events and dose modifications with lenalidomide in combintion with dexamethasone (abstract 3708). ASH Annual Meeting Abstracts Blood, 2008.

[20] Kyle RA, Pierre RV, Bayrd ED. Multiple myeloma and acute myelomonocytic leukemia. N Engl J Med, 283: 1121-5, 1970.

[21] Cuzick J, Erskine S, Edelman D, Galton DA. A comparison of the incidence of the myelodysplastic syndrome and acute myeloid leukaemia following melphalan and cyclophosphamide treatment for myelomatosis. A report to the Medical Research Council's working party on leukaemia in adults. Br J Cancer, 55: 523-9, 1987.

[22] Dores GM, Cote TR, Travis LB. New Malignanacies Following Hodgkin Lymphoma, Non-Hodgkin Lymphoma, and Myeloma. In: Curtis R, Freedman D, Ron E, Ries R, Hacker D, Edwards G, et al., editors. New Malignancies Among Cancer Survivors: SEER Cancer Registries, 1973-2000. Bethesda, MD: National Cancer Institute; 2006.

[23] Attal M, Cances Lauwers V, Marit G, et al. Lenalidomide maintenance after autologous transplantation for myeloma: a prospective randomized study of the Intergroupe Francophone du Myelome (IFM 2005-02 trial). 13th Biennial International Myeloma Workshop, Paris, France, 3-6 May 2011.

[24] Palumbo A, Bringhen S, Zweegman S, et al. Secondary malignancy in elderly myeloma patients. 13th Biennial International Myeloma Workshop, Paris, France, 3-6 May 2011.

[25] McCarthy P. A Phase III randomized, double-blind study of maintenance therapy with lenalidomide (CC 5013) or placebo following autologous stem cell transplantation for multiple myeloma. 13th Biennial International Myeloma Workshop, Paris, France, 3-6 May 2011.

[26] Dimopoulos M, Weber D, Richardson P, et al. Lenalidomide and dexamethasone (LEN + DEX) treatment in relapsed/refractory multiple myeloma patients does not increase the risk of second primary malignancies (SPM): Analysis of MM-009/010. 16th Congress of the European Hematology Association (EHA), London, England, 9-12 June 2011. 